\title{
Perception of the end position of a limb loaded with a weight
}

\author{
Hiroshi Shibata $\cdot$ Jiro Gyoba $\cdot$ Yasuhiro Takeshima
}

Published online: 9 November 2011

(C) Psychonomic Society, Inc. 2011

\begin{abstract}
We examined the effect of loading a weight on the perception of the end position of the limb. The participants haptically perceived where the end of their limb was located while they swung the limb or statically held it. The results showed that the loading of a weight influenced participants' perception only when they swung the limb; when the weight was attached to a participant's hand, the position was perceived to be farther from the body than when the weight was attached to the forearm or no weight was attached. We also found that the end position was generally underestimated when the participants swung their limb. On the other hand, when the participants statically held their limb, the perceived position was not affected by the loading of the weight and was rather precise. These results suggest that the perceptual system for limb end position is sensitive to changes in the surrounding haptic information caused by moving the limb.
\end{abstract}

Keywords Dynamic touch · Haptic perception · Perception and action

The ability to haptically perceive the position of our body parts is very important in daily life. For example, it helps us control our body parts smoothly without visual information. The haptic perception of body position occurs through mechanical stimulation of the mechanoreceptors (e.g., muscle spindles and Golgi tendon organs) present in our muscles and tendons.

It has been reported that vibrations sent to muscles and tendons (de Vignemont, Ehrsson, \& Haggard, 2005; Lackner, 1988; Longo, Kammers, Gomi, Tsakiris, \&

H. Shibata $(\bowtie) \cdot J$. Gyoba $\cdot$ Y. Takeshima

Graduate School of Arts \& Letters, Tohoku University,

Kawauchi 27-1, Aoba-ku,

Sendai 980-8576, Japan

e-mail: hshibata@sal.tohoku.ac.jp
Haggard, 2009) can change the spatial perception of the size of a body part, as can anesthetization of a finger (Gandevia \& Phegan, 1999). It is also possible to perceive sensations for body configurations that we have never experienced before (e.g., the Pinocchio illusion; Lackner, 1988). It can therefore be assumed that in addition to stored knowledge on body shape, information regarding online haptic stimulus changes is important in perceiving the shapes and spatial dimensions of our body (Pagano \& Turvey, 1998).

It has been hypothesized that common information is used for the haptic perception of spatial dimensions of both body segments and rod-shaped objects because both are basically cylindrical in shape and the haptic perception of both is caused by the stimulation of mechanoreceptors (Pagano \& Turvey, 1998; Turvey, 1996). For example, if the body segment from shoulder to fingertip is considered a rod, the length perception of the limb will be similar to that of a handheld rod.

The perception of the properties of handheld objects based on the stimulation of mechanoreceptors is called dynamic touch (Turvey, 1996). Many studies have investigated the length perception of a grasped rod by dynamic touch (e.g., Burton \& Turvey, 1990; Carello, Santana, \& Burton, 1996; Chan, 1994; Kingma, Beek, \& van Dieën, 2002; Kingma, van de Langenberg, \& Beek, 2004; Menger \& Withagen, 2009; Solomon \& Turvey, 1988; Stephen \& Arzamarski, 2009; Stroop, Turvey, Fitzpatrick, \& Carello, 2000; van de Langenberg, Kingma, \& Beek, 2006). In the typical task used in these studies, participants had to perceive the far end position of a grasped rod while the rod was occluded from view by a screen. The manipulations in the studies were based on the resistance of the rods and took into account both rod mass and rod mass distribution. The length perception of a grasped rod 
changes depending on the manipulation of the mass distribution, even if the mass and rod length remain the same. For example, when a weight is attached to the rod farther away from the rotational point (e.g., the wrist), the perceived rod length is longer (i.e., the perceived end position is farther) than when the weight is attached closer to the rotational point, because resistance to rotational accelerations is larger in the former case. It has been found that length perception when a rod is wielded is related to the moment of inertia and static moment, while length perception when a rod is held is related to the static moment; however, these findings are not consistent across studies. $^{1}$

We hypothesized that if a similar mechanism is involved in the length perception (or end position perception) of a body and a rod, the perception of where the end of the limb is located can be influenced by manipulating the mass distribution of the limb - for example, by loading a weight onto the limb. Some studies have demonstrated that haptic perception of limb direction is dependent on the manipulation of mass distribution (Pagano, Garrett, \& Turvey, 1996; Pagano \& Turvey, 1995; van de Langenberg, Kingma, \& Beck, 2007, 2008). Other studies have shown that tool use can influence our body representation as if our hand extended to the tip of the tool (e.g., Berti \& Frassinetti, 2000; Farnè \& Lādavas, 2000; Iriki, Tanaka, \& Iwamura, 1996; Longo \& Lourenco, 2006; Maravita, Husain, Clarke, \& Driver, 2001; Maravita \& Iriki, 2004; Maravita, Spence, Kennett, \& Driver, 2002). In the present study, we examined how loading a weight affected participants' perception of their limb's end position while the limb was occluded from view.

It should be noted that there are a few differences between the end position perception of a limb and end

\footnotetext{
${ }^{1}$ Basically, the moment of inertia and static moment are calculated by $\mathbf{\Sigma} \mathbf{m} \mathbf{r}^{2}$ and $\boldsymbol{\Sigma} \mathbf{m r}$, respectively (e.g., Stroop et al., 2000; Turvey, 1996), where $\mathbf{r}$ is the position vector of a small element of mass $\mathbf{m}$. In the calculation of the moment of inertia of a rod that is grasped, for example, the mass of each component mass particle of the grasped rod is multiplied by the square of its distance from the wrist. In addition, when a rod is wielded in a three-dimensional space, the inertia tensor (a symmetrical $3 \times 3$ matrix with six independent values) is thought to be related to the rod length perception, and the first eigenvalue of the inertia tensor (the largest resistance) and the third eigenvalue of the inertia tensor (the smallest resistance) are frequently manipulated in the task of rod length perception (for a review, see Turvey, 1996). However, some researchers have obtained different findings. Recent studies have found that length perception when a rod is wielded is implicated with not only the first eigenvalue of the inertia tensor, but also the static moment (Kingma, van de Langenberg, \& Beek, 2004); rod length perceptions are based on the most salient moments, depending on mechanical contexts (e.g., rod orientation and exploration style) (van de Langenberg et al., 2006); and feedback, mechanical contexts, and individual differences are related to the determination of what physical characteristics are used (Menger \& Withagen, 2009).
}

position perception of a rod. We humans already possess knowledge of our own body shape and size; we can, therefore, use this knowledge to estimate the end position of our own limbs, even if we cannot access online haptic information. On the other hand, when we haptically perceive the far end of a rod that we have grasped for the first time, we must use online haptic information, because we do not have prior knowledge of the rod's length. In this study, we modified the typical task used in the perception of a rod's far end position. Specifically, we changed the participants' elbow position on each trial, instead of changing the length of the handheld rod, and we manipulated the far end position of the participants' limb. We also asked the participants to move or hold the limb for a specific amount of time and in specific ways (i.e., range, direction, and tempo, which we determined on the basis of our pilot study), because they might be able to perceive their limb's end position without relying on changes in online haptic information. For the manipulation of the mass distribution of the participants' limbs, we changed the position of an attached weight (the weight was attached to their hand or forearm). We also conducted the experiment in the no-weight condition, in addition to the weightattached conditions. We took into consideration the possibility that the participants would feel their unweighted limb to be the usual and normal state and that their perception of the weighted limb could be affected by the perception of the unweighted limb if these conditions were used in the same experiment (e.g., they could refer to the haptic information of their unweighted limb when perceiving their weighted limb). In order to avoid this contamination, we first conducted separate experiments with and without weights and then examined the influence of the perception of the unweighted limb by conducting an additional experiment. We investigated the participants' perceptions of their limb's end position when they swung the limb and when they statically held the limb in a specific position. After the participants completed the task, we asked them to answer a questionnaire about the change in their perceived limb length during the task. In addition to examining their end position perception, we investigated whether or not the loading of a weight affected the participants' subjective perception of changes in limb length.

\section{Experiment 1A}

In Experiment 1A, the participants perceived their limb's end position after they swung the limb with a weight attached to the hand or forearm. We investigated whether the position of the weight affected their perception of the limb's end position. 


\section{Method}

Participants Ten right-handed males (mean age $=22.5$ years; standard deviation $[S D]=2.3$ years) participated in this experiment. Owing to the heaviness of the weight $(2 \mathrm{~kg})$, we recruited male participants. The mean length from the right elbow to the tip of the right middle finger was $45.7 \mathrm{~cm}(S D=1.1)$. All the participants were unaware of the aims of this study, and none participated in the other experiments included in this study.

Apparatus The apparatus used for this experiment is shown in Fig. 1. A chair was fixed to the floor, and a chinrest was used such that the participant's posture was restricted but his ability to move the right forearm was retained. An elbow rest was used; it could be set at four positions at intervals of $3 \mathrm{~cm}$. The upper part of the elbow rest was made of sponge and had a concave shape to accommodate the elbow; additional sponges were attached on both sides to restrict sideways movement of the participant's arm.

A rigid board was placed between the participant's face and right arm. A line marker at $0^{\circ}$ was attached at elbow height on the right side of the board. In addition, 20 angle line markers were prepared to indicate $-10^{\circ}, 10^{\circ}, 15^{\circ}$, and $45^{\circ}$ angles at each of the four potential elbow positions. The $-10^{\circ}$ and $10^{\circ}$ angle lines were used to indicate the permissible lower range $\left(0^{\circ} \pm 10^{\circ}\right)$, and the $15^{\circ}$ and $45^{\circ}$ angle lines were used to indicate the permissible upper range $\left(30^{\circ} \pm 15^{\circ}\right)$. We recorded each participant's swing motions with a digital video camera (15 frames/s) and crosschecked the motions with the angle line markers.

A scale was used to indicate the perceived end position of the limb to the precise millimeter. The numbers on the scale were moved horizontally for each trial in order to prevent the participants from relying on a fixed number. The board was $90 \mathrm{~cm}$ wide. The numbers that were presented ranged from $20 \mathrm{~cm}$ to $1 \mathrm{~m} 10 \mathrm{~cm}$, from $35 \mathrm{~cm}$ to $1 \mathrm{~m} 25 \mathrm{~cm}$, and so on. A metronome set to $1 \mathrm{~Hz}$ was placed to the right of the participant to indicate the frequency and timing for the arm swing.
The weight we used was a fitness-style cuff weight made of nylon; it contained 10 metal cylinders placed in individual pockets that were parallel to one another. These metal cylinders were $9 \mathrm{~cm}$ in length and $1.8 \mathrm{~cm}$ in diameter, with a weight of $200 \mathrm{~g}$ each; the total weight of the tool was thus approximately $2 \mathrm{~kg}$. This cuff weight was fastened around the participant's hand or forearm with a Velcro strap in such a way that five of the cylinders were on each side of the participant's hand or forearm, parallel to the fingers and the forearm (i.e., when the weight was on the hand, five cylinders were on the palmar side and five were on the dorsal side of the hand).

Procedure Each participant sat on the chair with his chin placed on the chinrest. The height of the chinrest was adjusted so that the participant was comfortable. The participant was asked to remain as still as possible. An experimenter sat at the participant's right side to manipulate the position of the elbow rest, the participant's arm, and the weight. Before the experimental trials, the experimenter verbally instructed the participant about the required swing angle $\left(0^{\circ}-30^{\circ}\right)$ and frequency $(1$ movement/s) and demonstrated the requirements by moving the participant's forearm. This instruction was conducted for each weight condition (e.g., with the weight attached separately to the forearm and to the hand). The elbow positions were the near and far positions (as shown in Fig. 1). The participant swung his weighted limb until he was able to do so appropriately without the experimenter's support. After the instruction practice, one practice trial per experimental condition was conducted (eight practice trials in all). If a participant's swing motion deviated from the permissible angles, the experimenter provided the participant with verbal guidance and asked him to perform the swings again. After the practice trials were completed, the experimental trials commenced.

First, each participant was instructed to wait with his eyes closed and with both hands placed under the table on his knees. On receiving a verbal cue from the experimenter, each participant lifted his right hand from under the table.
Fig. 1 Experimental apparatus used in Experiments 1A, 1B, $2 \mathrm{~A}, 2 \mathrm{~B}$, and 3
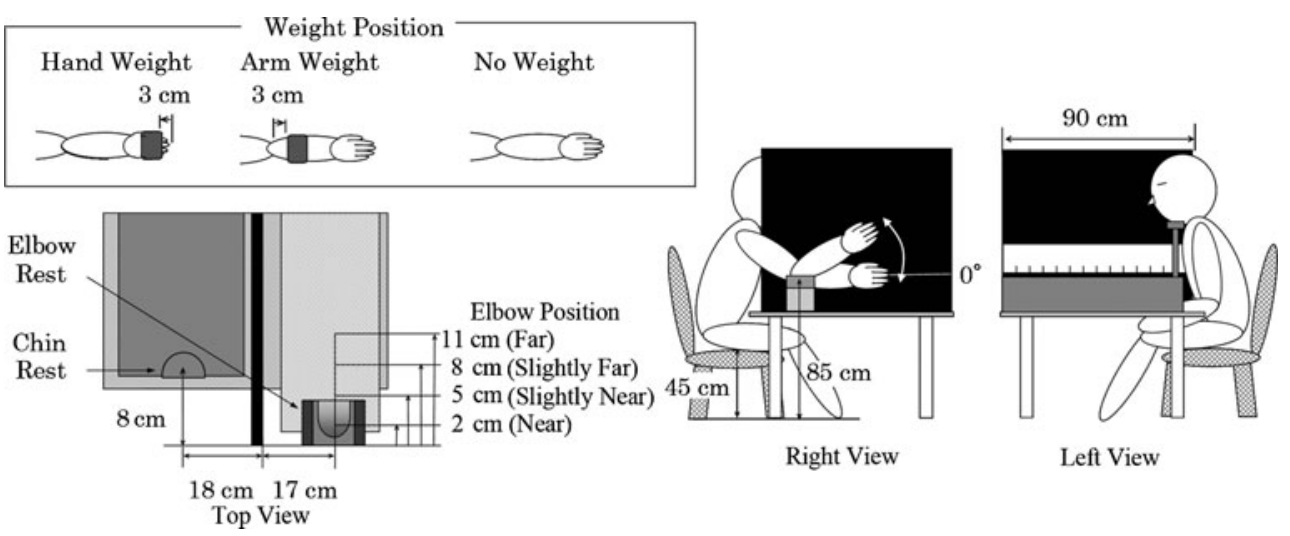

Left View 
The experimenter lifted the participant's arm, placed the elbow on the elbow rest, and held the forearm in a vertical position. The positions used for the elbow rest were near, slightly near, slightly far, and far (see Fig. 1 for details). The experimenter attached a weight to the participant's hand or forearm, moved the forearm, and held it at an angle of $30^{\circ}$. The experimenter turned on the metronome, gave a verbal cue, and removed his hand from the participant's forearm. The participant then opened his eyes and, within $5 \mathrm{~s}$, began moving his forearm. After the participant had moved his forearm 10 times (time, $10 \mathrm{~s}$; frequency, 1 movement/s [five swings in all]; angle, $0^{\circ}-30^{\circ}$ ), the experimenter turned off the metronome, and the participant verbally indicated a number on the scale to report the position of the end of his middle finger. If the perceived end position of the limb changed during the 10 -s period, the participant reported the final position (the position after the fifth swing). The participant was asked to place his right hand on his knee immediately after finishing the swing segment and was also asked to close his eyes immediately after reporting the number. The next trial started after the experimenter moved the numbers on the scale to a new position. If the participant's swing motion deviated from the permissible angles, the experimenter verbally instructed the participant to modify the swing before the next trial.

The experiment comprised four blocks, and each block comprised eight conditions (two weight positions $\times$ four elbow positions) in random order. Short rest intervals were taken between blocks. We conducted a total of 32 trials. The participants could not see their limbs during either the practice or the experimental trials.

After all the trials were completed, each participant was asked whether his perception of the length of his right limb changed during the limb swing. The participant was asked to answer a questionnaire and to rate his response for each weight condition on a 13-point rating scale $(-6=$ very short compared to the usual limb length; $0=$ no change; and $6=$ very long) ${ }^{2}$ After the participant answered the questionnaire, the experimenter measured the distance between the participant's elbow and middle fingertip on the right hand to calculate the actual end position of the limb at each elbow position.

Analysis and checking of motion A person who was unaware of the purpose of this study coded the video clips on which the participants' forearm motions were recorded. He determined whether the tip of the middle finger deviated from the permitted angles $\left(0^{\circ} \pm 10^{\circ}\right.$ and $\left.30^{\circ} \pm 15^{\circ}\right)$ during the 10 movements (five swings) for each trial, and he

\footnotetext{
${ }^{2}$ We also asked the participants about the change in their perception of limb length for the near and far elbow positions. However, the participants could not correctly recognize the difference and numbers of the elbow position; therefore, we will not discuss these data.
}

counted the number of deviated movements. The means of the deviated movements across all participants were as follows: no deviation, 28.8 trials $(90.0 \%, S D=2.6)$; one deviation, 1.9 trials $(5.9 \%, S D=1.7)$; and two to four deviations, 1.3 trials $(4.1 \%, S D=1.4)$.

\section{Results and discussion}

We calculated the perceived end position of the limb in comparison with the actual end position for each condition and participant. The mean perceived end position $(S D)$ for each condition was as follows: hand near, $-3.1 \mathrm{~cm}(5.8)$; hand slightly near, $-4.2 \mathrm{~cm}(5.9)$; hand slightly far, $-5.6 \mathrm{~cm}$ (6.2); hand far, $-6.8 \mathrm{~cm}$ (6.4); arm near, $-4.3 \mathrm{~cm}$ (5.2); arm slightly near, $-5.2 \mathrm{~cm}(6.3)$; arm slightly far, $-7.3 \mathrm{~cm}(6.3)$; and arm far, $-7.9 \mathrm{~cm}$ (6.6) (see Fig. 2). A two-way repeated measures analysis of variance (ANOVA) was conducted using the factors of weight position (hand vs. arm weight) and elbow position (near, slightly near, slightly far, and far). There were significant main effects for the weight position, $F(1,9)=9.46, p=.013, \eta_{\mathrm{p}}^{2}=.51$, and for the elbow position, $F(3,27)=38.27, p<.001, \eta_{\mathrm{p}}^{2}=.81$. The interaction between weight position and elbow position was not significant, $F(3,27)=1.01, p=.40, \eta_{\mathrm{p}}^{2}=.10$. In addition, we compared the perceived limb end position of each condition with 0 to investigate the tendency of the participants to underestimate the position. The perceived position was significantly different from 0 in the following three conditions: hand far, $t(9)=3.38, d=1.07$; arm slightly far, $t(9)=3.66, d=1.16$; and arm far, $t(9)=3.76$, $d=1.19(p<.05$, corrected $){ }^{3}$ The perceived position was not significantly different from 0 in the other five conditions: hand near, $t(9)=1.67, d=0.53$; hand slightly near, $t(9)=2.24 ; d=0.71$; hand slightly far, $t(9)=2.88, d=$ 0.91 ; arm near, $t(9)=2.57, d=0.81$ arm slightly near, $t(9)=2.62, d=0.83$ ( $p>.05$, corrected).

Figure 3 shows the mean score for the questionnaire on the perception of limb length change for each weightposition condition. The mean score $(S D)$ for each condition was as follows: hand-weight condition, 2.4 (1.4); armweight condition, -1.6 (1.4). The score in the hand-weight condition was significantly higher than that of the armweight condition, $t(9)=5.07, p<.001, d=2.80$. The score in the hand-weight condition was significantly higher than $0, t(9)=5.31, d=1.68$, and that of the arm-weight condition was significantly lower than $0, t(9)=3.54, d=$ $1.12(p<.05$, corrected).

These results showed that manipulation of the weight position affected the perception of where the end of the

\footnotetext{
${ }^{3}$ The $p$ values were corrected for multiple comparisons using the sequentially rejective Bonferroni test.
} 
Fig. 2 Mean differences between the perceived and actual end positions for each condition in Experiments 1A (hand-weight and arm-weight conditions) and $1 \mathrm{~B}$ (no-weight condition). A negative value shows underestimation. Error bars represent standard errors

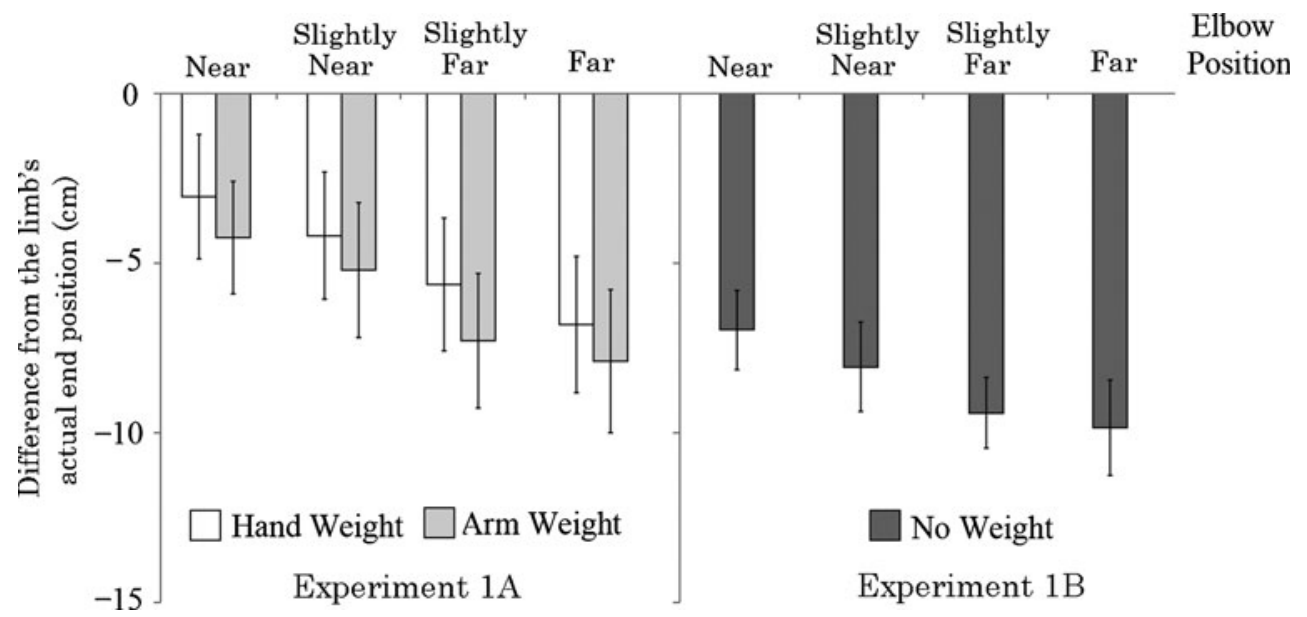

limb was located. The end position in the hand-weight condition was perceived to be farther than the end position in the arm-weight condition. This result is consistent with that of other studies that have investigated the far-endposition perception of a rod (e.g., Solomon \& Turvey, 1988). In addition, the questionnaire results showed that manipulation of the weight position also affected the participants' subjective perception of limb length change.

We also found that manipulation of elbow position affected the perception of limb end position. In this experiment, we manipulated elbow position in order to change the actual end position of the limb on each trial. Therefore, we did not predict the effect of elbow position, but there was a general observation that the greater the distance of the elbow from the body, the more likely the participant was to underestimate the limb's end position. Indeed, there was a general tendency for the limb's end position to be underestimated, although significant underestimation was not observed when the participant's elbow was placed near to the body. On the other hand, the results

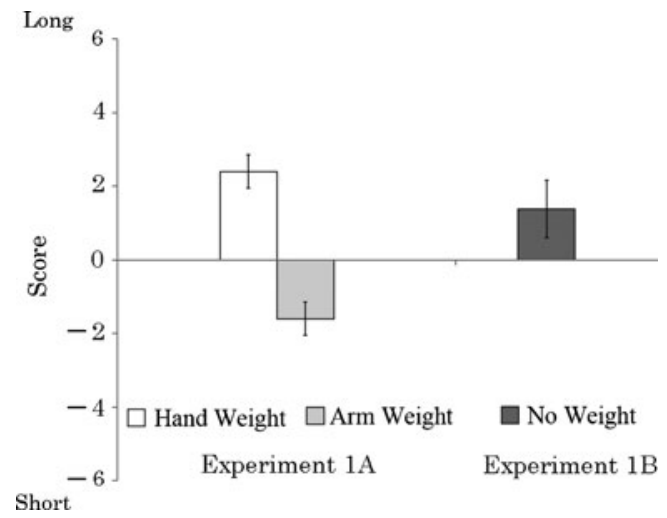

Fig. 3 Mean scores in the questionnaire on the subjective perception of limb-length change for each condition in Experiments 1A (handweight and arm-weight conditions) and $1 \mathrm{~B}$ (no-weight condition). A high score indicates the perception that limb length is greater than the usual limb length, and a low score indicates the perception of a shorter limb. Error bars represent standard errors of the questionnaire showed that the participants perceived limb length to be long in the hand-weight condition. These results suggest that swinging a limb to which a weight was attached decreased the accuracy of the end position perception; the results also suggest that participants were unaware of their inaccurate perceptions.

\section{Experiment 1B}

The results of Experiment $1 \mathrm{~A}$ showed that the manipulations of weight position and elbow position affected the participants' perception of their limb's end position. We also found a general tendency for the end position to be underestimated. It is possible that irrespective of manipulations to the weight position, an attached weight causes the perception that the limb's end position is closer to the body. In Experiment 1B, the participants estimated end position after swinging their limb without an attached weight. The aim of this experiment was to investigate the effect of elbow position and examine participants' tendency to underestimate the end position in the no-weight condition.

Method

Participants Eight right-handed males (mean age $[S D]=$ 23.5 [2.8] years) participated in this experiment. The mean length from the right elbow to the tip of the right middle finger was $45.0 \mathrm{~cm}(S D=2.2)$. All the participants were unaware of the aims of this study and did not participate in the other experiments of this study.

Apparatus The apparatus was the same as that used in Experiment 1A, except that the weight was not used (see Fig. 1).

Procedure Each participant was asked to perceive his limb's end position after swinging the forearm without a 
weight attached to it. Before the experimental trials, the experimenter instructed each participant on how to swing his forearm, and 4 practice trials were conducted. The experiment comprised four blocks, and each block comprised four conditions (four elbow positions). A short rest interval was taken after the second block was completed. We conducted a total of 16 trials. With the exception of these differences, all the procedures were identical to those used in Experiment 1A.

Analysis and checking of motion A person unaware of the purpose of this study coded the video clips as to whether the tip of the participant's middle finger deviated from the permitted angles $\left(0^{\circ} \pm 10^{\circ}\right.$ and $\left.30^{\circ} \pm 15^{\circ}\right)$ in the 10 movements for each trial. The means of deviated movements across all the participants were as follows: no deviation, 15.3 trials $(95.3 \%, S D=1.2)$; one deviation, 0.3 trials $(1.6 \%, S D=0.5)$, and two deviations, 0.5 trials $(3.1 \%, S D=1.1)$.

Results and discussion

The mean perceived end position $(S D)$ for each condition was as follows: near, $-7.0 \mathrm{~cm}(3.3)$; slightly near, $-8.1 \mathrm{~cm}$ (3.7); slightly far, $-9.4 \mathrm{~cm}$ (3.0); far, ${ }^{4}-9.8 \mathrm{~cm} \mathrm{(4.0)}$ (see Fig. 2). A one-way repeated measures ANOVA with the factor of elbow position was conducted. The main effect of elbow position was significant, $F(3,21)=19.77, p<.001$, $\eta_{\mathrm{p}}{ }^{2}=.74$. The perceived position was significantly different from 0 in all the conditions: near, $t(7)=5.97, d=2.11$; slightly near, $t(7)=6.09, d=2.15$; slightly far, $t(7)=9.02$, $d=3.19$; and far, $t(7)=7.01, d=2.48(p<.05$, corrected $)$.

We then compared Experiments $1 \mathrm{~A}$ and 1B; we tested the hand-weight condition versus the no-weight condition and the arm-weight condition versus the no-weight condition by using a two-way mixed ANOVA with the factors of weight condition and elbow position for each comparison. The results showed no significant difference between the end positions in the hand-weight and no-weight conditions; the main effect of the weight condition and the interaction were not significant, $F(1,16)=2.32, p=.15, \eta_{\mathrm{p}}{ }^{2}=.13$, and $F(3,48)=0.95, p=.42, \eta_{\mathrm{p}}{ }^{2}=.06$, respectively. We also found no significant difference between end positions in the arm-weight and no-weight conditions; the main effect of the weight condition and the interaction were not signifi-

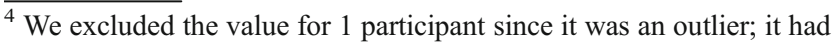
a difference of more than $18.6 \mathrm{~cm}$ from the values of the other three trials in the same condition. We included this participant's data in the analysis of this experiment because the other values were normal and the dispersion of each condition was not large (the difference between the two extreme values in each condition was within $3.3 \mathrm{~cm}$ ) after excluding the outlier.
}

cant, $F(1,16)=1.00, p=.33, \eta_{\mathrm{p}}{ }^{2}=.06$, and $F(3,48)=$ $1.00, p=.40, \eta_{\mathrm{p}}^{2}=.06$, respectively.

Figure 3 shows the mean score for the questionnaire on perceived limb length when the participants swung the limb with no weight attached $(M=1.4[S D=2.2])$. There was no significant difference in the score from $0, t(7)=1.77, p=.12$, $d=0.63$. We then tested the difference between the weight and no-weight conditions. There was no significant difference between the scores in the hand-weight and no-weight conditions, $t(16)=0.81, d=0.55(p>.05$, corrected). On the other hand, there was significant difference between the scores in the arm-weight and no-weight conditions, $t(16)=$ $3.13, d=1.60$ ( $p<.05$, corrected).

Consistent with the results of Experiment 1A, this experiment showed that the end position was underestimated and that the manipulation of elbow position affected the perception of the end position. These results showed that the effect of elbow position and the participants' tendency to underestimate were not due to the loading of a weight. In this experiment, the end position was highly underestimated, although the comparison between Experiments $1 \mathrm{~A}$ and $1 \mathrm{~B}$ did not show a significant difference between end position perception in the no-weight and weight conditions. The questionnaire results revealed that the score in the no-weight condition was not significantly different from 0 (in addition, it was higher than that in the arm-weight condition). These findings suggest that, despite their strong tendency to underestimate, the participants did not have a subjective perception of short limb length.

\section{Experiment 2A}

In Experiment 2A, the participants estimated their limb's end position after they held their forearm horizontal with a weight attached to the hand or forearm. We investigated whether the effect of loading a weight would also be found in this static condition.

\section{Method}

Participants Eight right-handed males (mean age $=23.6$ $[S D=4.1]$ years) participated in this experiment. The mean length from the right elbow to the tip of the right middle finger was $46.0 \mathrm{~cm}(S D=1.8)$. All the participants were unaware of the aims of this study and did not participate in the other experiments of this study.

Apparatus The apparatus was the same as that used in Experiment 1A (see Fig. 1).

Procedure Each participant was asked to perceive his limb's end position after holding his arm horizontal with a 
weight attached to it. Before the experimental trials, the experimenter instructed each participant on how to hold the forearm. In the practice and experimental trials, the experimenter attached the weight to the participant's hand or forearm and then held the forearm at an angle of $0^{\circ}$. After the experimenter let go of the participant's forearm, the participant was required to hold the forearm in that position for $10 \mathrm{~s}$, which was sounded by the metronome (set at $1 \mathrm{~Hz}$ ). With the exception of these differences, the procedures were identical to those in Experiment 1A.

Analysis and checking of forearm holding A person unaware of the aims of the study coded the video clips as to whether the tip of the participants' middle finger deviated from the permitted angle $\left(0^{\circ} \pm 10^{\circ}\right)$ during the time the participants held his forearm in the static position (10 s). All the participants correctly held the limb within the permitted angle in all the trials.

Results and discussion

The mean perceived end position $(S D)$ for each condition was as follows: hand near, $-0.3 \mathrm{~cm}(6.8)$; hand slightly near, $-1.3 \mathrm{~cm}$ (7.0); hand slightly far, $-3.0 \mathrm{~cm}$ (6.9); hand far, $-3.9 \mathrm{~cm}$ (7.2); arm near, $-0.6 \mathrm{~cm}$ (6.5); arm slightly near, $-1.4 \mathrm{~cm}$ (7.0); arm slightly far, $-3.7 \mathrm{~cm}$ (7.0); and arm far, $-4.4 \mathrm{~cm} \mathrm{(7.1)} \mathrm{(see} \mathrm{Fig.} \mathrm{4).} \mathrm{A} \mathrm{two-way} \mathrm{repeated}$ measures ANOVA was conducted by using the factors of weight position and elbow position. The main effect of elbow position was significant, $F(3,21)=58.49, p<.001$, $\eta_{\mathrm{p}}{ }^{2}=.89$. There were no significant differences for the main effect of weight position, $F(1,7)=1.47, p=.27, \eta_{\mathrm{p}}{ }^{2}=$ .17 , or for the interaction between weight position and elbow position, $F(3,21)=0.40, p=.76, \eta_{\mathrm{p}}{ }^{2}=.05$. Although participants had a tendency to underestimate, the perceived end position in all the conditions was not significantly different from 0 : hand near, $t(7)=0.12, d=$ 0.04 ; hand slightly near, $t(7)=0.53, d=0.19$; hand slightly far, $t(7)=1.23, d=0.43$; hand far, $t(7)=1.51, d=0.53$; arm near, $t(7)=0.26, d=0.09$; arm slightly near, $t(7)=$ $0.58, d=0.20$; arm slightly far, $t(7)=1.51, d=0.53$; and arm far, $t(7)=1.77, d=0.62(p<.05$, corrected $)$.

Figure 5 shows the mean score for the questionnaire on participants' limb length perception for each weight position (condition). The mean score $(S D)$ for each condition was as follows: hand-weight condition, 1.0 (3.3); arm-weight condition, -0.6 (1.5). There was no significant difference between the two weight conditions, $t(7)=0.98, p=.36, d=$ 0.64 . The score for each condition was not significantly different from 0 : hand weight, $t(7)=0.86, d=0.30$; arm weight, $t(7)=1.17, d=0.42(p>.05$, corrected $)$.

The results showed that when the participants held the limb in a static position, a change in the position of the weight did not affect their perceptions of the end position or limb length. In addition, we found that the participants' perceptions of their limb's end position were quite precise when they held it in one position. Consistent with the results of Experiments $1 \mathrm{~A}$ and $1 \mathrm{~B}$, changes in elbow position affected the participants' perception of end position.

\section{Experiment 2B}

In Experiment 2A, it was shown that the perception of end position during static positioning of a limb was quite precise and that the tendency to underestimate the end position was slight. It is possible that loading a weight during static limb positioning may cause the end position to be perceived as farther from the body. In Experiment 2B, we investigated the participants' tendency to underestimate the end position under the no-weight condition.

\section{Method}

Participants Eight right-handed males (mean age $=21.6$ $[S D=2.4]$ years) participated in this experiment. The mean
Fig. 4 Mean differences between the perceived and actual end positions for each condition in Experiments 2A (hand-weight and arm-weight conditions) and 2B (no-weight condition). A negative value shows underestimation. Error bars represent standard errors

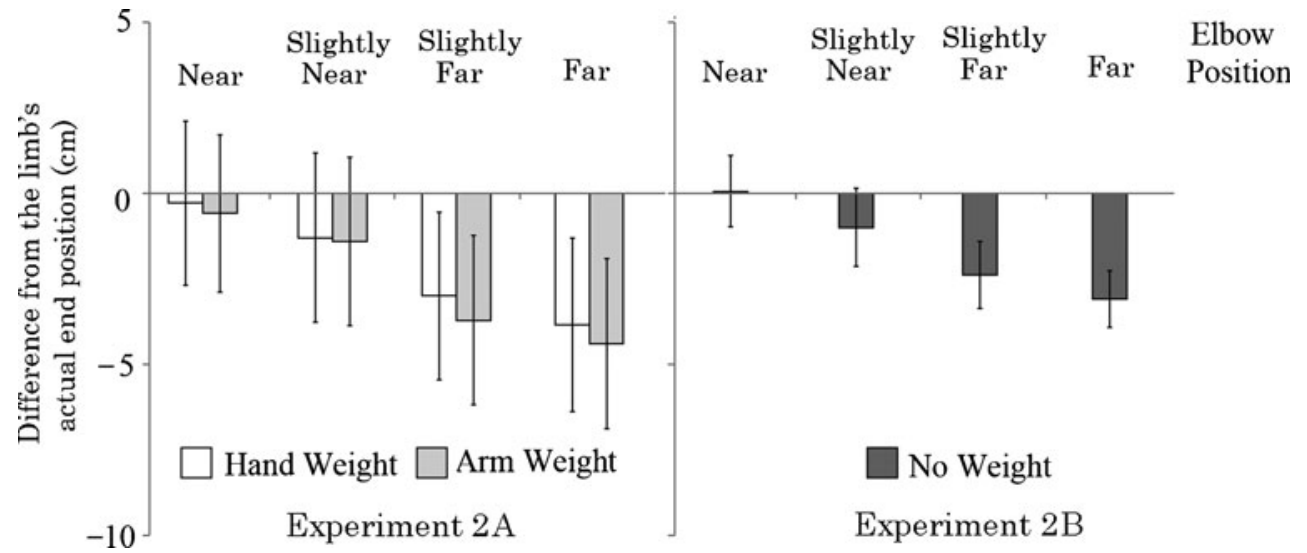




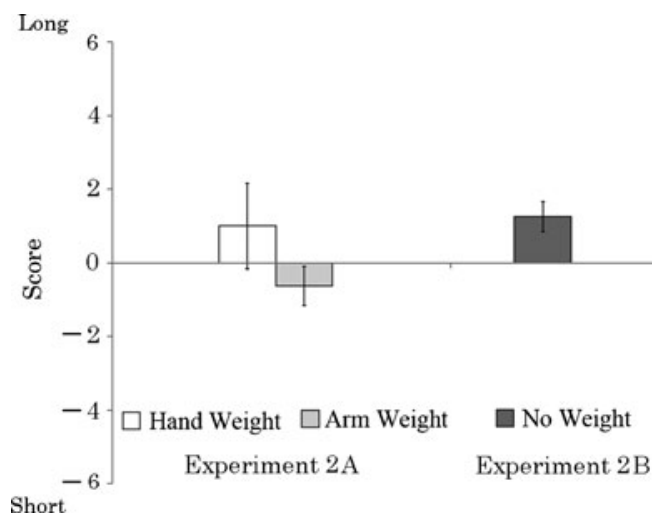

Fig. 5 Mean scores in the questionnaire on the subjective perception of limb length change for each condition in Experiments 2A (handweight and arm-weight conditions) and 2B (no-weight condition). A high score indicates the perception that limb length is greater than the usual limb length, and a low score indicates the perception of a shorter limb. Error bars represent standard errors

length from the right elbow to the tip of the right middle finger was $45.7 \mathrm{~cm}(S D=1.5)$. All the participants were unaware of the aims of this study and did not participate in the other experiments of this study.

Apparatus The apparatus was the same as that in Experiment 1A, except that the weight was not used (see Fig. 1).

Procedure Each participant was asked to perceive his limb's end position after holding his forearm horizontal with no weight attached. Before the experimental trials, the experimenter instructed each participant on how to hold the forearm (with no weight used). Four practice trials were conducted. The experiment comprised four blocks, and each block comprised four conditions (four elbow positions). A short rest interval was taken after the second block was completed. We conducted a total of 16 trials. With the exception of these differences, the procedures were identical to those used in Experiment $2 \mathrm{~A}$.

Analysis and checking of forearm holding A person unaware of the purpose of this study coded the video clips as to whether the tip of the participant's middle finger deviated from the permitted angle $\left(0^{\circ} \pm 10^{\circ}\right)$ during the time the participant held his forearm in the static position (10 s). All the participants correctly held the limb within the permitted angle on all the trials.

Results and discussion

The mean perceived end position for each condition was as follows: hand near, $0.1 \mathrm{~cm}(3.0)$; hand slightly near, $-1.0 \mathrm{~cm}$ (3.2); hand slightly far, $-2.4 \mathrm{~cm}$ (2.8); hand far, $-3.1 \mathrm{~cm}(2.3)$ (see Fig. 4). A one-way repeated measures ANOVA with the factor of elbow position was conducted. The main effect of elbow position was significant, $F(3,21)=22.94$, $p<.001, \eta_{\mathrm{p}}{ }^{2}=.77$.

Although a tendency to underestimate was observed, the perceived end position was significantly different from 0 only in the far condition, $t(7)=3.73, d=1.32(p<.05$, corrected). The perceived position was not significantly different from 0 in the other three conditions: near, $t(7)=.05, d=0.02$; slightly near, $t(4)=.88, d=0.31$; and slightly far, $t(7)=2.46$, $d=0.87(p>.05$, corrected).

We then compared Experiments $2 \mathrm{~A}$ and 2B; we tested the hand-weight condition versus the no-weight condition and the arm-weight condition versus the no-weight condition by using a two-way mixed ANOVA with the factors of weight condition and elbow position for each comparison. The results showed no significant difference between the end position in the hand-weight and no-weight conditions; the main effects of the weight condition and the interaction were not significant, $F(1,14)=0.04, p=.85, \eta_{\mathrm{p}}{ }^{2}=.003$, and $F(3,42)=0.28, p=.84, \eta_{\mathrm{p}}{ }^{2}=.02$, respectively. We also found no significant difference between the end position in the arm-weight and no-weight conditions; the main effects of the weight condition and the interaction were not significant, $F(1,14)=0.13, p=.73, \eta_{\mathrm{p}}{ }^{2}=.01$, and $F(3,42)=1.11, p=.36, \eta_{\mathrm{p}}{ }^{2}=.07$, respectively.

Figure 5 shows the mean score for the questionnaire on the perception of limb length $(M=1.3[S D=1.2])$. There was a significant difference between that score and $0, t(7)=3.03, p=.019, d=1.07$. We then tested the difference between the weight and no-weight conditions. There was no significant difference between the scores in the hand-weight and no-weight conditions, $t(14)=0.20$, $d=0.10$ ( $p>.05$, corrected). On the other hand, there was a significant difference between the scores in the armweight and no-weight conditions, $t(14)=2.79, d=1.39$ $(p<.05$, corrected $)$.

These results showed that the perception of end position during static positioning of the limb was quite precise, even with no weight attached to the limb. Consistent with the results of the other experiments, changing the elbow position affected the participants' perceptions. The comparison between Experiments $2 \mathrm{~A}$ and $2 \mathrm{~B}$ did not show a significant difference between the perceived end position in the no-weight and weight conditions. The results of the questionnaire showed that the participants subjectively perceived the length of their unweighted limb to be greater than their usual limb length (although the score was not very high), and the score of the unweighted limb was higher than that of the arm-weighted limb. There may be a tendency for an unweighted limb to be subjectively perceived as slightly longer when the limb is held horizontally. 


\section{Experiment 3}

The four experiments above showed that the loading of a weight influenced participants' position perception only when they swung their limb. However, the comparison between Experiments 1A and 1B did not show a significant difference; there were no significant differences in the perceived end position between the hand-weight and noweight conditions or between the arm-weight and no-weight conditions. Resistance to rotational accelerations of the perceivers' limb was larger in the weighted limb condition than in the unweighted limb condition; therefore, if the perceived end position depends on changes in resistance to rotational accelerations, it is predicted that the end position of the weighted limb would be perceived to be farther than that of the unweighted limb. Indeed, Solomon and Turvey (1988) have shown that the end position of a handheld rod attached with a weight was perceived to be farther than that of the rod without the weight.

One reason that a significant difference was not observed between Experiments 1A and 1B could be the factor of individual differences (i.e., large standard deviation within each weight condition). We conducted the weight and noweight conditions in separate experiments because we took into consideration the possibility that the perception of the unweighted limb affects the perception of the weighted limb. Although we did not find a significant difference between the hand-weight and no-weight conditions, the mean values of Experiments $1 \mathrm{~A}$ and $1 \mathrm{~B}$ seemed to show a systematic increase depending on the change in resistance to rotational accelerations. The mean values of the perceived end position in the no-weight condition were closest to the rotational position (i.e., the elbow), and those in the hand-weight condition were farthest if these were compared with respect to the same elbow position.

We then conducted an additional experiment as Experiment 3 , wherein a within-subjects comparison was adopted. The purpose of the experiment was to investigate the difference of the end position perception between the weight condition (the hand-weight condition) and the no-weight condition more directly.

Method

Participants Nine right-handed males (mean age $=20.3$ $[S D=1.4]$ years) participated in this experiment. The mean length from the right elbow to the tip of the right middle finger was $45.7 \mathrm{~cm}(S D=1.9)$. All the participants were unaware of the aims of this study and did not participate in the other experiments of this study.

Apparatus The apparatus was the same as that used in Experiment 1A (see Fig. 1).
Procedure Each participant was asked to perceive his limb's end position after swinging his limb with a weight attached to his hand or without the weight. With the exception of this difference, the procedures were identical to those in Experiment 1A.

Analysis and checking of motion A person unaware of the purpose of this study coded the video clips as to whether the tip of the participant's middle finger deviated from the permitted angles $\left(0^{\circ} \pm 10^{\circ}\right.$ and $\left.30^{\circ} \pm 15^{\circ}\right)$ in the 10 movements for each trial. The means of deviated movements across all the participants were as follows: no deviation, 27.7 trials $(86.5 \%, S D=4.3)$; one deviation, 3.1 trials $(9.7 \%, S D=2.8)$, and two deviations, 1.2 trials $(3.8 \%, S D=2.0)$.

\section{Results and discussion}

The mean perceived end position $(S D)$ for each condition

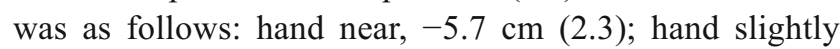
near, $-7.6 \mathrm{~cm}$ (2.7); hand slightly far, $-8.9 \mathrm{~cm} \mathrm{(2.6)}$; hand far, $-10.6 \mathrm{~cm} \mathrm{(2.6);} \mathrm{no-weight} \mathrm{near,}-6.1 \mathrm{~cm}$ (2.6); noweight slightly near, $-7.7 \mathrm{~cm}(2.2)$; no-weight slightly far,

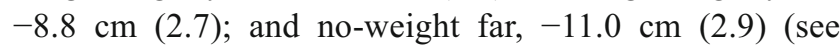
Fig. 6). A two-way ANOVA was conducted using the factors of weight condition and elbow position. There were significant main effects for the weight condition, $F$ $(1,8)=7.12, p=.028, \eta_{\mathrm{p}}{ }^{2}=.47$, and for the elbow position, $F(3,24)=80.45, p<.001, \eta_{\mathrm{p}}^{2}=.91$. The interaction was not significant, $F(3,24)=0.77, p=.52$, $\eta_{\mathrm{p}}{ }^{2}=.08$. The perceived position was significantly different from 0 in all the conditions: hand near, $t(8)=$ $7.35, d=2.45$; hand slightly near, $t(8)=8.31, d=2.77$; hand slightly far, $t(8)=10.21, d=3.40$; hand far, $t(8)=$ $12.19, d=4.06$; no-weight near, $t(8)=6.86, d=2.29$; no-

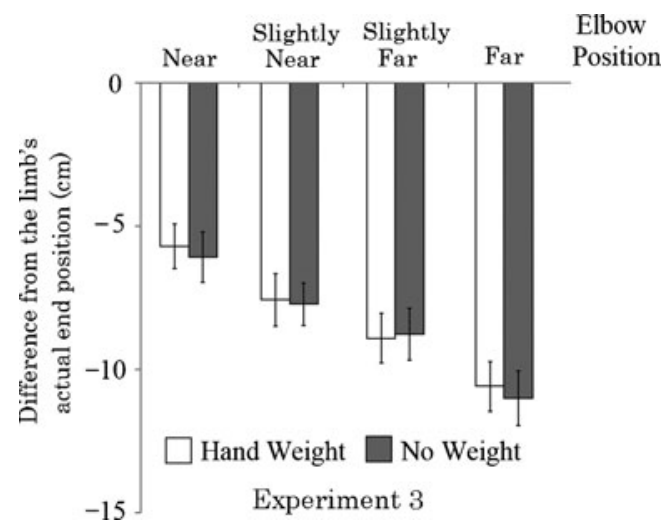

Fig. 6 Mean differences between the perceived and actual end positions for each condition in Experiments 3 (hand-weight and noweight conditions). A negative value shows underestimation. Error bars represent standard errors 
weight slightly near, $t(8)=10.52, d=3.51$; no-weight slightly far, $t(8)=9.72, d=3.24$; no-weight far, $t(8)=$ $11.42, d=3.81(p<.05$, corrected).

Figure 7 shows the mean score for the questionnaire on the perception of limb length change for each weight condition. The mean score $(S D)$ for each condition was as follows: hand-weight condition, -0.7 (2.5); no-weight condition, 1.0 (2.0). There was no significant difference between the two weight conditions, $t(8)=1.15, p=.28, d=0.74$. The score for each condition was not significantly different from 0 : hand-weight condition, $t(8)=0.80, d=0.27$; no-weight condition, $t(8)=1.50, d=0.50(p>.05$, corrected $)$.

These results showed that the manipulation of the weight affected the end position perception; the end position in the hand-weight condition was perceived to be farther than that in the no-weight condition, although the difference between the two conditions was very small. On the other hand, the questionnaire results did not show a significant difference between the two conditions. Consistent with the results of Experiments $1 \mathrm{~A}$ and 1B, a strong underestimation of the end position was observed in this experiment. The effect of the elbow position was also observed.

\section{General discussion}

In this study, we conducted five experiments wherein the participants perceived their limb's end position when the limb was occluded from view by a board. We found that the participants' end position perception while swinging their limbs changed according to the manipulation of the weight loaded to their limbs.

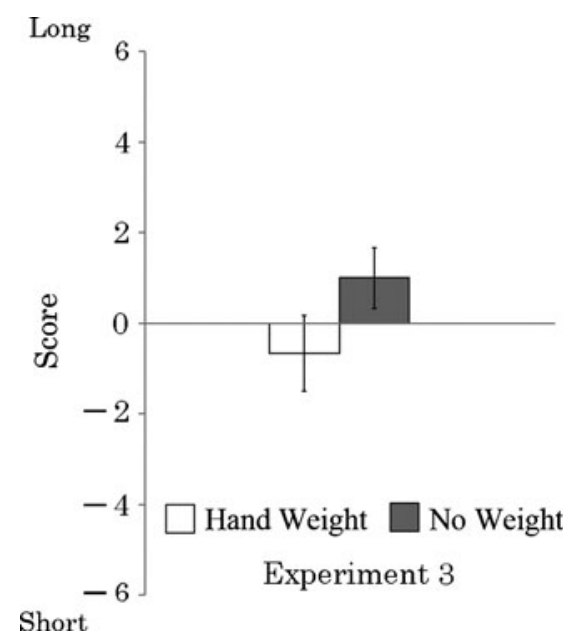

Fig. 7 Mean scores in the questionnaire on the subjective perception of limb length change for each condition in Experiment 3 (handweight and no-weight conditions). A high score indicates the perception that limb length is greater than the usual limb length, and a low score indicates the perception of a shorter limb. Error bars represent standard errors
Effect of loading a weight when the limb is swung

The results of Experiment 1A showed that the perceived limb's end position while the limb was swung changed depending on the position of the attached weight. When the weight was attached to the hand, the participants perceived the end position to be more distant than when the weight was attached to the forearm. The important points derived from the results of Experiment 1A are as follows: (1) The difference in haptic information that occurs with a change in the weight's position (e.g., an increase or decrease in resistance to rotational accelerations, such as the moment of inertia) is interpreted as a change in the limb's end position, and (2) the change in the pattern corresponds to the results of the far-end-position perception of a handheld rod (i.e., in common with the perception of a handheld rod; when a weight is attached farther away from the rotational point, the end position is perceived as farther). These results might be attributed to the difficulty in differentiating the haptic information contributed only by the weight from the haptic information contributed by the weight and the limb (i.e., the participants might be unable to subtract the former from the latter, probably because the information is the same or similar for both). The results of our study are consistent with the hypothesis that a common mechanism is involved in the haptic perception of the spatial dimensions of a limb and a handheld object (Pagano \& Turvey, 1998; Turvey, 1996). Although further research is needed to identify precise haptic information, our results indicate that humans rely on information pertaining to the mass distribution of the limb when haptically perceiving their limbs' end position.

The results of Experiment 3 showed that the end position perception also changed by the manipulation with or without the weight; when the weight was attached to the hand, the participants perceived the end position to be more distant than when no weight was attached. Resistance to rotational accelerations of the perceivers' limbs was larger in the hand-weight condition than in the no-weight condition. Therefore, this result also supports the hypothesis that a similar mechanism is involved in the end position perception of a body and a rod. However, the difference between the hand-weight and no-weight conditions (i.e., the results in Experiment 3) was very small, as compared with the difference between the hand-weight and arm-weight conditions (i.e., the results in Experiment 1A). In Experiment 1A, we did not include the no-weight condition because there was a possibility that the perception of the unweighted limb affects the perception of the weighted limb. Actually, the perceivers in Experiment 3 could use the end position perception in the no-weight condition as a reference when they perceived the position of their weighted limb. This may be related to the small 
difference between the hand-weight and no-weight conditions in Experiment 3.

Difference between loading a weight when the limb is swung and when it is held statically

In contrast to the results of Experiment 1, the results of Experiment 2 did not show an effect for loading a weight. The change in the weight position did not affect the perception of end position, and the perceptions in the two weight conditions (Experiment 2A) and the no-weight condition (Experiment 2B) were similar. These results suggest that when the limb is held in a static position, the mechanism used to perceive the end position does not make use of changes in haptic information contributed by the weight (e.g., increase or decrease in the magnitude of the static moment).

Humans are assumed to perceive dynamic changes in stimuli through active movements (e.g., active touch) and to obtain useful information for spatial perception (e.g., Gibson, 1962, 1966). Of course, in the static holding condition, stimulus change in the mechanoreceptors is assumed to be involved because motor adjustment would be required to maintain the same position, but this change is thought to be much larger when the forearm is swung. Therefore, spatial perception with active movements (such as limb swinging) might be sensitive to a change in haptic information.

Another difference between the perceptual mechanism involved in the swinging and statically holding conditions is the function of efference copy. When we produce voluntary movements, it is assumed that we predict sensory feedback via the function involving the efference copy of the motor command (e.g., Blakemore, Wolpert, \& Frith, 1998, 2000; Christensen et al., 2007; Wolpert \& Flanagan, 2001). In addition, some studies have discussed heaviness perceptions in an expectation model (Davis \& Brickett, 1977; Davis \& Roberts, 1976; Loomis, 1907; Ross \& Gregory, 1970; Woodworth, 1921). This model assumes that when we move an object, we predict how much force is required, and the discrepancy of predicted movement and actual afferent signal is associated with the size-weight illusion, in which a large object is perceived as lighter than smaller objects of the same mass. For example, this illusion is explained as follows: Perceivers expect that greater force is required to move a large object, as compared with a small object of the same mass, but if the object moves more than the expected amount with the applied force, they perceive the object as lighter, although Amazeen and Turvey (1996) have reported that heaviness perception depends on rotational inertia.

In the present study, the participants would predict the feedback of the haptic information via the function involving the efference copy of the motor command of their limb swings. Therefore, predicted haptic feedback, in addition to the actual haptic signals, would be involved in the perception of the end position. In this case, there is a possibility that more intensive haptic feedback would be predicted via the motor command of the swinging movement of the hand-weighted limb, as compared with that of the arm-weighted limb (because of greater moment of inertia in the former case), even if the efference copies of the two weight conditions are the same (although the efference copies actually would not be the same). Therefore, differences in the predicted sensory feedback, depending on the manipulation of the weight loaded to the limb, may be involved in producing the change in the endposition perceptions.

On the other hand, in the limb-holding condition, the influence of predicted haptic feedback may be relatively weak. Although the actual haptic information is assumed to be more intensive in the hand-weight condition than in the arm-weight condition (because of greater static moment in the former case), the dynamic change in predicted haptic feedback via the motor command to maintain the position is thought to be quite small. In addition, it is believed that there is little discrepancy between predicted haptic feedback and actual haptic information while the limb is statically held (on the other hand, the discrepancy would occur to some extent when a weighted limb is swung). This dynamic change in predicted sensory feedback via the function of efference copy might be related to the difference between the perceived end positions of a swinging limb and a statically held limb.

Underestimation when the limb is swung and when it is statically held

We found a general tendency to underestimate the end position when the participants swung their limb. However, the underestimation was very weak when the participants held their limb in a static position. It has been reported that vibrations sent to muscle-tendon units generate the illusion of position and movement of the limb (Goodwin, McCloskey, \& Matthews, 1972; Lackner, 1988) and that the simultaneous vibration of muscle-tendon units in the biceps and triceps in an immobilized limb (i.e., conflicting illusions of elbow extension and flexion) induces a contraction of the limb representation (Longo et al., 2009). The underestimation during the movement of the limb might be related to the stimulation of muscle-tendon units in the biceps and triceps caused by the limb swings. Another interpretation could pertain to the mass distribution of an unweighted limb. The mass of a limb (from the elbow to the fingertip) is mainly distributed around the elbow. If the perception of the end position depends on the characteristics of resistance to 
rotational accelerations, the relatively small mass of body parts located far from the rotational point (the relatively small mass of fingers in this case) may be associated with the underestimation of the end position.

Previous studies, however, have already reported that the end position (e.g., fingertip position or the end position of a fisted hand) is underestimated to some extent in static and no-weight conditions (Fuentes \& Bastian, 2010; Longo et al., 2009; McDonnell, Scott, Dickison, Theriault, \& Wood, 1989; Wann \& Ibrahim, 1992). Humans would have a general tendency to underestimate the end position of the limb, even when it is statically held.

\section{Subjective perception of limb length change}

It should be noted that the type of body representation is related to changes in the perception of the end position of a limb. Previous studies have reported that body representations can be broken down into at least three distinct types: body semantics (lexical-semantic representations such as the names and functions of body parts), body structural descriptions (visuospatial representations that define the topological locations and boundaries of body parts), and body schema (dynamical representations of the relative positions of one's own body parts) (Buxbaum \& Coslett, 2001; Corradi-Dell'Acqua \& Rumiati, 2007; Schwoebel \& Coslett, 2005; Sirigu, Grafman, Bressler, \& Sunderland, 1991). It is assumed that body schema is derived from motor and multiple sensory inputs such as proprioception; the online position perceptions are related to body schema.

Previous studies (de Vignemont et al., 2005; Lackner, 1988; Longo et al., 2009) have reported that vibrations sent to muscles change the perception of body size in addition to the perception of body posture. These results suggest that the online perception of body positions (or body schema) is involved not only with the perception of body postures, but also with the perception of body sizes such as limb length. In the present study, in addition to end position perceptions, we examined changes in the participants' subjective perception of their limb length change after conducting the task. The results of the questionnaire in Experiments 1 and 2 showed that participants' perceived that limb length in the hand-weight condition was longer than that in the arm-weight condition only when they swung the limb. This change pattern was consistent with the results of the perceived end position of the limb; the perceived end position in the hand-weight condition was farther from the body than that in the arm-weight condition only when they swung the limb. These results suggest that the change in haptic and proprioceptive information based on the position of the attached weight affects limb length perception, and the change in the perceived end position is caused by the change in the perceived limb length.
On the other hand, the results of the questionnaire in Experiment 3 were not consistent with those of the perceived end position. The perceived end position in the hand-weight condition was farther from the body than that in the no-weight condition; however, the questionnaire scores did not show a significant difference between the two conditions. This suggests that the perceivers in Experiment 3 did not have the subjective image that their weighted limb was longer, as compared with their unweighted limb, in spite of the significant difference of the perceived end position between the two conditions. Here, it must be noted that there is a potential contrast with the results of recent studies of near-space perception. On the basis of results of line bisection tasks, Longo and Lourenco $(2006,2007)$ reported that there was a systematic relationship between limb length and the size of near space and that tool use expanded the size of near space. They also found that the size of near space shrank when weights were attached to their participants' wrists, as compared with the size of near space with unweighted limbs (Lourenco \& Longo, 2009). Moreover, they suggested that there may be a relationship between the size of near space and perceived limb length, although they did not directly measure the perception of the fingertip position or the perception of limb length. Rather, their participants manipulated a tripod with a laser pointer to indicate the midpoint of a line. Therefore, two contrast factors may be involved in our study. The first is the increase in resistance to rotational accelerations when a weight is attached to the limb. This could be related to the perception of the end position being farther from the rotational position. The second is the decrease in the size of near space with a weighted limb, which may be associated with the subjective perception of the end position being closer to the rotational position. It may be necessary to consider these two influences in interpreting the inconsistency in results between the end position perception and the subjective perception of limb length change in Experiment 3.

Moreover, the results showing underestimation of the end position of the limb were not consistent with the results of the questionnaire. The scores in Experiments 1B and 3 showed that the participants did not subjectively perceive the limb to be short, even though they highly underestimated the limb's end position. These results suggest that the underestimation of the end position while the limb is swung is not related to the subjective perception of the limb length as short, and the participants were not aware that they underestimated the end position of the limb.

Influence of the change in elbow position

In this study, we manipulated the participants' elbow position to change their fingertip position, but we found 
that the greater the distance of the elbow position from the body, the more likely it was that the participants would underestimate the limb's end position. These results suggest that the degree of underestimation was not constant but, rather, changed depending on the participants' postures and that the end position could be determined from the relation between the fingertip and other body parts, such as the relative distance between the fingertip and body trunk. Strong underestimation with an extended limb posture might be useful in some situations-for example, when an object is grasped-because there is a small margin beyond the estimated distance. Indeed, there are some studies that have shown that estimated reachabilities are related to postural changes (e.g., Fischer, 2000; Robinovitch, 1998).

Recent studies have reported that the proprioceptive position sense is more precise when the perceiver's hand is closer to the body (Wilson, Wong, \& Gribble, 2010) or when there is a weak correlation between the degree of error of the perceived fingertip position and the distance from the fingertip to other body parts (head and shoulder) (Fuentes \& Bastian, 2010). However, these studies did not report that fingertip position is generally underestimated depending on relative distance from fingertip to other body parts. In the present study, one possible reason that underestimation was found to be dependent on the participants' posture could be related to the experimental procedure of the study. In the previous studies, the participants reported their proprioceptive position on a two-dimensional scale (i.e., on a plane horizontal to the floor). However, in the present study, the participants reported the perceived end position of their limb on a one-dimensional scale. In addition, we did not inform the participants that their elbow position was moved parallel to the board between the head and the right arm (we instructed them only that the elbow position changed for each trial). Therefore, even if the participants perceived that the angle of the right limb deviated from the line parallel to the board and/or the floor, the present study could not measure these deviations. If these deviations are large when the elbow is placed far from the body, the underestimation may be strong according to the deviations. Additional experiments using a two- or three-dimensional scale would help clarify the effect of elbow position on fingertip perception. Another reason may be retention of the previous trial's proprioception. Different from the previous studies (Fuentes \& Bastian, 2010; Wilson, et al., 2010), the present study asked the participants to set their right hand on their knee after performing each trial. The cancellation effect of the previous trial's proprioception would be greater in the present study. This cancellation might be associated with the underestimation, depending on the participants' posture.

\section{Conclusion}

This study revealed that loading a weight to the limb influenced the perception of where the end of the limb was located, but only when the participants swung their limbs. These results suggest that the perceptual system for judging the end position of a limb is sensitive to changes in the haptic information of the loaded limb caused by moving the limb.

Author Note We thank Professor M. Turvey for his very helpful comments regarding an earlier version of the manuscript. We are also very grateful to anonymous reviewers and the action editor for their valuable and insightful comments and suggestions. This study was supported by the 2005 Satow's Research Fund for Behavioral Science and by Grants-in-Aid for JSPS Fellows to the first author (Nos. 184952 and 20-2537).

\section{References}

Amazeen, E. L., \& Turvey, M. T. (1996). Weight perception and the haptic size-weight illusion are functions of the inertia tensor. Journal of Experimental Psychology: Human Perception and Performance, 22, 213-232.

Berti, A., \& Frassinetti, F. (2000). When far becomes near: Remapping of space by tool use. Journal of Cognitive Neuroscience, 12, 415420.

Blakemore, S.-J., Wolpert, D. M., \& Frith, C. D. (1998). Central cancellation of self-produced tickle sensation. Nature Neuroscience, 1, 635-640.

Blakemore, S.-J., Wolpert, D. M., \& Frith, C. D. (2000). Why can't you tickle yourself? NeuroReport, 11, R11-R16.

Burton, G., \& Turvey, M. T. (1990). Perceiving the lengths of rods that are held but not wielded. Ecological Psychology, 2, 295324.

Buxbaum, L. J., \& Coslett, H. B. (2001). Specialised structural descrpitions for human body parts: Evidence from autotopagnosia. Cognitive Neuropsychology, 18, 289-306.

Carello, C., Santana, M.-V., \& Burton, G. (1996). Selective perception by dynamic touch. Perception \& Psychophysics, 58, 1177-1190.

Chan, T.-C. (1994). Haptic perception of partial-rod lengths with the rod held stationary or wielded. Perception \& Psychophysics, 55, 551-561.

Christensen, M. S., Lundbye-Jensen, J., Geertsen, S. S., Petersen, T. H., Paulson, O. B., \& Nielsen, J. B. (2007). Premotor cortex modulate somatosensory cortex during voluntary movements without proprioceptive feedback. Nature Neuroscience, 10, 417 419.

Corradi-Dell'Acqua, C., \& Rumiati, R. I. (2007). What the brain knows about the body: Evidence for dissociable representations. In F. Santoianni \& C. Sabatano (Eds.), Brain development in learning environments: Embodied and perceptual advancements (pp. 50-64). Newcastle: Cambridge Scholars Publishing.

Davis, C. M., \& Brickett, P. (1977). The role of preparatory muscluar tension in the size-weight illusion. Perception \& Psychophysics, $22,262-264$.

Davis, C. M., \& Roberts, W. (1976). Lifting movements in the sizeweight illusion. Perception \& Psychophysics, 20, 33-36.

de Vignemont, F., Ehrsson, H. H., \& Haggard, P. (2005). Bodily illusions modulate tactile perception. Current Biology, 15, 12861290. 
Farnè, A., \& Lādavas, E. (2000). Dynamic size-change of hand peripersonal space following tool use. NeuroReport, 11, 16451649.

Fischer, M. H. (2000). Estimating reachability: Whole body engagement or postural stability? Human Movement Science, 19, $297-$ 318.

Fuentes, C. T., \& Bastian, A. J. (2010). Where is your arm? Variations in proprioception across space and tasks. Journal of Neurophysiology, 103, 164-171.

Gandevia, S. C., \& Phegan, C. M. L. (1999). Perceptual distortions of the human body image produced by local anaesthesia, pain and cutaneous stimulation. The Journal of Physiology, 514, 609-616.

Gibson, J. J. (1962). Observations on active touch. Psychological Review, 69, 477-491.

Gibson, J. J. (1966). The senses considered as perceptual systems. Boston: Houghton Mifflin.

Goodwin, G. M., McCloskey, D. I., \& Matthews, P. B. C. (1972). Proprioceptive illusions induced by muscle vibration: Contribution by muscle spindles to perception? Science, 175, 1382-1384.

Iriki, A., Tanaka, M., \& Iwamura, Y. (1996). Coding of modified body schema during tool use by macaque postcentral neurons. NeuroReport, 7, 2325-2330.

Kingma, I., Beek, P. J., \& van Dieën, J. H. (2002). The inertia tensor versus static moment and mass in perceiving length and heaviness of hand-wielded rods. Journal of Experimental Psychology: Human Perception and Performance, 28, 180-191.

Kingma, I., van de Langenberg, R., \& Beek, P. J. (2004). Which mechanical invariants are associated with the perception of length and heaviness of a nonvisible handheld rod? Testing the inertia tensor hypothesis. Journal of Experimental Psychology: Human Perception and Performance, 30, 346-354.

Lackner, J. R. (1988). Some proprioceptive influences on the perceptual representation of body shape and orientation. Brain, 111, 281-297.

Longo, M. R., Kammers, M. P. M., Gomi, H., Tsakiris, M., \& Haggard, P. (2009). Contraction of body representation induced by proprioceptive conflict. Current Biology, 19, R727-R728.

Longo, M. R., \& Lourenco, S. F. (2006). On the nature of near space: Effects of tool use and the transition to far space. Neuropsychologia, 44, 977-981.

Longo, M. R., \& Lourenco, S. F. (2007). Space perception and body morphology: Extent of near space scales with arm length. Experimental Brain Research, 177, 285-290.

Loomis, H. N. (1907). Reactions to equal weights of unequal size. Psychological Monographs, 8, 334-348.

Lourenco, S. F., \& Long, M. R. (2009). The plasticity of near space: Evidence for contraction. Cognition, 112, 451-456.

Maravita, A., Husain, M., Clarke, K., \& Driver, J. (2001). Reaching with a tool extends visual-tactile interactions into far space: Evidence from cross-modal extinction. Neuropsychologia, 39, 580-585.

Maravita, A., \& Iriki, A. (2004). Tools for the body (schema). Trends in Cognitive Sciences, 8, 79-86.

Maravita, A., Spence, C., Kennett, S., \& Driver, J. (2002). Tool-use changes multimodal spatial interactions between vision and touch in normal humans. Cognition, 83, B25-B34.

McDonnell, P. M., Scott, R. N., Dickison, J., Theriault, R. A., \& Wood, B. (1989). Do artificial limbs become part of the user? New evidence. Journal of Rehabilitation Research and Development, $26,17-24$.
Menger, R., \& Withagen, R. (2009). How mechanical context and feedback jointly determine the use of mechanical variables in length perception by dynamic touch. Attention, Perception, \& Psychophysics, 71, 1862-1875.

Pagano, C. C., Garrett, S. R., \& Turvey, M. T. (1996). Is limb proprioception a function of the limbs' inertial eigenvectors? Ecological Psychology, 8, 43-69.

Pagano, C. C., \& Turvey, M. T. (1995). The inertia tensor as a basis for the perception of limb orientation. Journal of Experimental Psychology: Human Perception and Performance, 21, 1070 1087.

Pagano, C. C., \& Turvey, M. T. (1998). Eigenvectors of the inertia tensor and perceiving the orientations of limbs and objects. Journal of Applied Biomechanics, 14, 331-359.

Robinovitch, S. N. (1998). Perception of postural limits during reaching. Journal of Motor Behavior, 30, 352-358.

Ross, H. E., \& Gregory, R. L. (1970). Weight illusions and weight discrimination: A revised hypothesis. Quarterly Journal of Experimental Psychology, 22, 318-328.

Schwoebel, J., \& Coslett, H. B. (2005). Evidence for multiple, distinct representations of the human body. Journal of Cognitive Neuroscience, 17, 543-553.

Sirigu, A., Grafman, J., Bressler, K., \& Sunderland, T. (1991). Multiple representations contribute to body knowledge processing. Brain, 114, 629-642.

Solomon, H. Y., \& Turvey, M. T. (1988). Haptically perceiving the distances reachable with hand-held objects. Journal of Experimental Psychology: Human Perception and Performance, 14, 404-427.

Stephen, D. G., \& Arzamarski, R. (2009). Self-training of dynamic touch: Striking improves judgment by wielding. Attention, Perception, \& Psychophysics, 71, 1717-1723.

Stroop, M., Turvey, M. T., Fitzpatrick, P., \& Carello, C. (2000). Inertia tensor and weight-percept models of length perception by static holding. Journal of Experimental Psychology: Human Perception and Performance, 26, 1133-1147.

Turvey, M. T. (1996). Dynamic touch. American Psychologist, 51, $1134-1152$.

van de Langenberg, R., Kingma, I., \& Beek, P. J. (2006). Mechanical invariants are implicated in dynamic touch as a function of their salience in the stimulus flow. Journal of Experimental Psychology: Human Perception and Performance, 32, 1093-1106.

van de Langenberg, R., Kingma, I., \& Beek, P. J. (2007). Perception of limb orientation in the vertical plane depends on center of mass rather than inertial eigenvectors. Experimental Brain Research, 180, 595-607.

van de Langenberg, R., Kingma, I., \& Beek, P. J. (2008). The perception of limb orientation depends on the center of mass. Journal of Experimental Psychology: Human Perception and Performance, 34, 624-639.

Wann, J. P., \& Ibrahim, S. F. (1992). Does limb proprioception drift? Experimental Brain Research, 91, 162-166.

Wilson, E. T., Wong, J., \& Gribble, P. L. (2010). Mapping proprioception across a 2D horizontal workspace. PLoS One, 5, e11851.

Wolpert, D. M., \& Flanagan, J. R. (2001). Motor prediction. Current Biology, 11, R729-R732.

Woodworth, R. S. (1921). Psychology: A study of mental life. New York: Holt. 